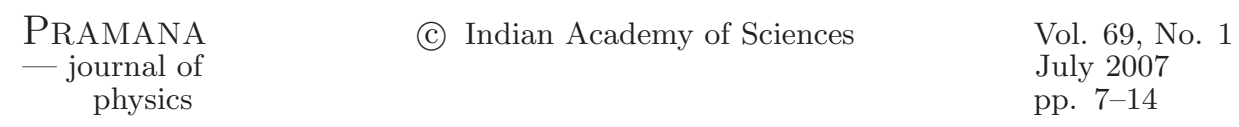

\title{
A K Raychaudhuri and his equation*
}

\author{
J EHLERS \\ Max-Planck-Institute for Gravitational Physics, Albert-Einstein-Insitute, \\ Am Mühlenberg 1, D-14476 Golm, Germany \\ E-mail: juergen.ehlers@aei.mpg.de
}

\begin{abstract}
Amal Kumar Raychaudhuri died on June 18, 2005. This essay follows the lecture which I gave in honour of this great Indian scientist and teacher on December 26, 2005 in Puri, India.
\end{abstract}

Keywords. Raychaudhuri equation; cosmology; gravitational collapse.

PACS Nos $01.60 ; 98.80$

\section{A K Raychaudhuri's scientific career}

AKR, as Raychaudhuri came to be called later by his colleagues and students, was born on September 14, 1923 in Barisal, in what is now Bangladesh. He studied at Presidency College, Kolkata, and obtained his B.Sc. and M.Sc. degrees in 1942 and 1944, respectively.

From 1945 to 1949 AKR worked as Research Assistant at the Indian Association for the Cultivation of Sciences (IACS) in Jadavpur, Kolkata. There, he had to work in experimental physics, which reflected neither his wishes nor his talent - he rather wanted to do research in mathematical physics, and he pursued this interest by studying general relativity on his own.

At that time general relativity was considered a difficult and useless subject, admitting no interaction between theory and experiment. So not only in India, but practically everywhere only few theoreticians pursued general relativity, among them were Pascual Jordan and Otto Heckmann, who from 1949 on were my teachers at the University of Hamburg.

In 1949, AKR got a temporary position as Lecturer of Physics at Asutosh College, where he wrote his third publication 'Arbitrary Concentrations of Matter and the Schwarzschild Singularity' [1]. In this Physical Review article he constructed an

\footnotetext{
*Invited plenary talk delivered at the International Conference on Einstein's Legacy in the New Millennium, December 15-22, 2005, Puri, India.
} 
exact model of a collapsing, spherical dust cloud surrounded by its vacuum gravitational field. Apparently unaware of the 1939 Oppenheimer-Snyder paper 'On Continued Gravitational Contraction', he solved the junction conditions required at the surface of the 'star' and showed that 'there is no theoretical limit to the degree of concentration, and the Schwarzschild singularity ... occurs only in some coordinate systems'. He contrasted his result with Einstein's model of circularly orbiting particles which, according to Einstein, establishes beyond reasonable doubt that the Schwarzschild singularity is physically unattainable, and he remarked that he had been led to this investigation by his doubts - no small achievement for a young self-made relativist.

In 1952 AKR returned to IACS, where he got a permanent position as Research Officer. There he had to work on solids, which resulted in three publications. He did not, however, neglect his main interest, turning his attention to structure formation in the universe and the question whether the Big Bang singularity of the cosmological models by Friedmann and Lemaitre will be present also in more general, nonsymmetric models, or can be circumvented. In trying to find an answer to this fundamental question, AKR found in 1953 what came to be called the Raychaudhuri equation, which will be discussed here. He submitted his important result to the Physical Review in December 1953, and it was published in 1955 [2]. Its importance and implications were however then not recognized by his Indian colleagues.

At this time exact solutions of Einstein's equations were discussed extensively at the Hamburg Seminar on General Relativity and Cosmology directed by Pascual Jordan. These efforts were closely related to the long-standing interest in cosmology which Otto Heckmann, Director of the Observatory in Bergedorf near Hamburg, pursued. The young members of the Jordan-Seminar (among them W Kundt, E Schucking, R K Sachs, M Truemper and I Ozsvath) were eager not just to review known solutions, but to find new ones, and to understand their intrinsic properties using newly developed mathematical methods like differential forms and moving frames. Good fortune had it that Engelbert Schucking happened to read AKR's article in the Physical Review and recognized its importance; he coined the name Raychaudhuri equation [3,4]. The Hamburg group applied and extended AKR's work, of which AKR learned through a letter, presumably from the young Indian student Bramachary, who was a member of the Jordan Seminar around 1956. AKR was very delighted about this recognition, which helped his standing in India a lot. It encouraged him to submit his work as a $\mathrm{PhD}$ thesis, and again, it was fortunate for AKR that John A Wheeler was chosen as his external examiner. Wheeler immediately recognized the significance of the work and saw to it that the $\mathrm{PhD}$ was granted, in 1960, 'with honours'. Promptly, in 1961, AKR was appointed

professor of physics at Presidency College, where he served until his retirement in 1988.

\section{The Raychaudhuri equation}

Around 1955, Raychaudhuri was interested mainly in two problems: 


\section{A K Raychaudhuri and his equation}

1. Is the Big Bang singularity due to the strong symmetry of the FriedmannLemaitre-Robertson-Walker models due to the strong symmetry assumptions which had been made when setting up these models?

2. Is a cosmological constant needed to solve the time-scale problem? Based on his discovery of different types of Cepheid variables, the astronomer Walter Baade had revised the extragalactic distance scale, and thereby ameliorated the time-scale problem. But was that sufficient?

AKR found his equation while searching for answers to these questions. I will now give a derivation of the equation.

Consider an arbitrary motion of a continuous medium in a general space-time $\left(M, g_{\alpha \beta}\right)$. Let the world lines of the fluid elements be generated by the 4 -velocity field $U$. Then the spatial tensors $\omega_{\alpha \beta}=\omega_{[\alpha \beta]}, \delta_{\alpha \beta}=\delta_{(\alpha \beta)}, \Theta=U_{; \alpha}^{\alpha}, h_{\alpha \beta}=$ $g_{\alpha \beta}+U_{\alpha} U_{\beta}$ which appear in the decomposition

$$
U_{\alpha \beta}=\omega_{\alpha \beta}+\sigma_{\alpha \beta}+\frac{1}{3} \Theta h_{\alpha \beta}-\dot{U}_{\alpha} U_{\beta}
$$

represent, respectively, the rate of rotation, shear, expansion, the spatial metric and the acceleration associated with the fluid motion. Insertion of eq. (1) into the equation

$$
U_{\alpha ;|\beta ; \gamma|}=\frac{1}{2} U_{\delta} R_{\alpha \beta \gamma}^{\delta}
$$

which defines the Riemann curvature tensor, gives the kinematical identity

$$
\dot{\Theta}+\frac{1}{3} \Theta^{2}=\dot{U}_{; \alpha}^{\alpha}+2\left(\omega^{2}-\sigma^{2}\right)-R_{\alpha \beta} U^{\alpha} U^{\beta},
$$

where the overdot indicates covariant differentiation along the world lines, e.g. $\dot{\Theta}=\Theta_{; \alpha} U^{\alpha}$. If eq. (2) is combined with Einstein's field equation

$$
R_{\alpha \beta}=\kappa\left(T_{\alpha \beta}-\frac{1}{2} T g_{\alpha \beta}\right)+\Lambda g_{\alpha \beta}
$$

and a length scale $l$ is introduced via the definition $l / l=\frac{1}{3} \Theta$, the (slightly generalized) Raychaudhuri equation

$$
3 \frac{\ddot{l}}{l}=\dot{U}_{; \alpha}^{\alpha}+2\left(\omega^{2}-\sigma^{2}\right)-\kappa\left(T_{\alpha \beta} U^{\alpha} U^{\beta}+\frac{1}{2} T\right)+\Lambda
$$

results. It is a covariant form of the (time, time) component, in a frame adapted to $U$, of the field equation (3). Equation (4) shows: If the acceleration term is nonpositive and, together with the shear and matter terms, dominates the rotation and $\Lambda$-terms, the mean motion is decelerating, otherwise accelerating.

In the original version of 1955, Raychaudhuri assumed pressureless matter of density $\rho$; then eq. (4) simplifies to

$$
3 \frac{\ddot{l}}{l}=-\frac{\kappa}{2} \rho-\sigma^{2}+\Lambda+2 \omega^{2} .
$$

Pramana - J. Phys., Vol. 69, No. 1, July 2007 
(The same relation holds in Newtonian theory if Poisson's equation is generalized to $\Delta \Phi=4 \pi \rho-\Lambda$.) Raychaudhuri drew several conclusions from his eq. (5):

(a) In the static case, eq. (5) reduces to the relation $\kappa / 2 \rho=\Lambda$ of Einstein's 'cylindrical' universe. AKR emphasises that in his approach that relation follows 'without any assumption regarding symmetry or conditions obtaining in distant parts of the universe', and he states (without proof) that 'the only static nonspinning universe is the Einstein universe'. This correct statement shows that spatial closure follows from staticity and local properties; it need not be assumed. (I remark in passing that an analogous characterization holds for Gödel's universe: It is the only dust solution of eq. (3) without shear, having covariantly constant rotation rate [5].)

(b) If $\omega=0$ on a world line and $\Lambda=0$, and if $i\left(t_{0}\right)>0$ for some $t_{0}$, then there exists a density singularity in the past of $t_{0}$, and the 'age' is bounded by $(l / i)_{0}$. This is the first singularity theorem without symmetry assumptions. The more general eq. (5) has been used, e.g., by Hawking-Ellis to argue for a hot Big Bang [6].

(c) If $\omega=0$ on a world line, then the time between a state $\left(\rho_{0},(i / l)_{0}\right)$ and a prior state with $l<l_{0}$, is maximal if $\sigma=0$.

(d) If a singularity happened on a world line, then the 'age' measured from the singularity increases with $\Lambda$ and $\omega$ and decreases with increasing $\rho$ and shear. 'Thus the longer time scales are due to an increased freedom in the choice of $\Lambda$ rather than to anisotropy'.

AKR's 1955 paper ends with the remark that in a dust model with vanishing rotation and shear the 3 -spaces orthogonal to the fluid world lines have constant curvature. (In fact, the space-time is then of the Robertson-Walker type [5].)

The paper reviewed here is truly a landmark in cosmology. It showed how results are in principle observable and local variables can be obtained without imposition of isometries. It can be considered a forerunner of a programme begun by J Kristian and R K Sachs and continued by G Ellis and his coworkers. Moreover, it paved the way to the Penrose-Hawking singularity theorems.

I should like to recall that R K Sachs in 1960 obtained equations similar to eq. (5) for congruences of light-like geodesics. They first played a part in gravitational radiation theory in connection with peeling theorems, and later and at present are employed in gravitational lensing, especially in locating caustics of optical wavefronts.

In his later work, AKR constructed several exact solutions, among them the one illustrating spherical, but inhomogeneous collapse in an expanding universe and space-times containing charged matter and electromagnetic fields. His monograph 'Theoretical Cosmology' [7], published in 1979 gives a comprehensive survey of the state-of-the-art at that time. The text distinguishes itself by the care with which assumptions and conclusions are stated and critically interpreted, and by the attention given to observations and alternative theoretical explanations thereof. The preface begins: 'The book aims to convey to the reader some of the excitement while not hiding the confusion that remains.' From 1990 onwards AKR became interested in singularity-free space-times and attempted to find properties that might characterize them.

AKR was the president of the Indian Association of General Relativity and Gravitation from 1978 to 1980 and Fellow of the Indian Academy of Sciences since 1982. 
Finally I would like to mention that Raychadhuri was not only a pathbreaking researcher, but also an outstanding teacher who created in many young minds enthusiasm for physics, especially general relativity and relativistic cosmology. $\mathrm{He}$ continued the line of great Indian physicists such as Saha, Raman, Bose, Bhabha and Chandrasekhar.

\section{List of Publications of Professor A K Raychaudhury}

\section{A. Papers}

1. Volkoff's massive spheres; Phys. Rev. 84, 166 (1951)

2. Radiation sphere in Einstein universe; Bull. Calcutta Math. Soc. 44, 31 (1952)

3. Condensations in expanding cosmological models; Phys. Rev. 86, 90 (1952)

4. Reine Strahlungsfelder mit Zentralsymmetrie in der allgemeinem Relativitätstheorie; Z. Physik. 135, 225 (1953)

5. Arbitrary concentration of matter and the Schwarzchild singularity; Phys. Rev. 89, 417 (1953)

6. Relativistic cosmology I; Phys. Rev. 98, 1123 (1955)

7. Note on the network approximation in metals; Proc. Phys. Soc. (London) A68, 439 (1955)

8. Perturbed cosmological models; Z. Astrophysik 37, 103 (1955)

9. Relativistic and Newtonian cosmology; Z. Astrophysik 43, 161 (1957)

10. Singular state in relativistic cosmology; Phys. Rev. 106, 172 (1957)

11. Electronic energy bands in model three dimensional lattices, Z. Physik 148, 435 (1957)

12. An anisotropic cosmological solution in general relativity; Proc. Phys. Soc. (London) A72, 263 (1958)

13. The isoelectronic series of semiconducting compounds with zinc-blende structure; Proc. Nat. Inst. Sci. (India) 25, 201 (1959)

14. Static electromagnetic fields in general relativity; Ann. Phys. (NY) 11, 501 (1960)

15. A general deduction of two important relations in relativistic cosmology; $Z$. Astrophysik 51, 88 (1961)

16. Stationary cylindrically symmetric clusters of particles in general relativity (with M M Som); Proc. Cambridge Philos. Soc. 58, 338 (1962)

17. Cosmological evolution with creation of matter (with S. Banerji); Z. Astrophysik 58, 187 (1964)

18. Gravitational collapse in a cosmological background; Proc. Phys. Soc. (London) $\mathbf{8 8 , 5 4 5 ( 1 9 6 6 )}$

19. Static distribution of charged dust in general relativity (with U K Deo); Proc. Roy. Soc. (London) A303, 97 (1968)

20. Stationary electromagnetic fields in general relativity (with B K Dutta); $J$. Math. Phys. 9, 1715 (1968) 
21. Cylindrically symmetric charged dust distribution in rigid rotation in general relativity (with M M Som); Proc. R. Soc. (London) A304, 81 (1968)

22. Charged dust distribution in general relativity (with U K De); J. Phys. A3, 263 (1970)

23. Tachyons and gravitation; J. Math. Phys. 15, 856 (1974)

24. Null fields in spaces of high symmetry (with A K Datta); J. Math. Phys. 15, 1277 (1974)

25. Spherically symmetric charged dust distribution in general relativity; Ann. Inst. Henri Poincare 22, 229 (1975)

26. Nature of the singularity in some Brans-Dicke universes; Prog. Theor. Phys. 53, $1360(1975)$

27. Einstein-Cartan cosmologies with a magnetic field; Phys. Rev. D12, 952 (1975)

28. Einstein-Cartan spheres (with S Banerji), Phys. Rev. D16, 281 (1977)

29. Perfect fluid cosmology with geodesic world lines (with S R Maity); Phys. Rev. D18, 3595 (1978)

30. Charged dust distribution in Brans-Dicke theory (with N Bandyopadhyay); Phys. Rev. D18, 2756 (1978)

31. Field of a charged particle in Brans-Dicke theory (with N Bandyopadhyay); Prog. Theor. Phys. 59, 414 (1978)

32. Ferraro's theorem in non-flat space-time; Mon. Not. R. Astron. Soc. 189, 39 (1979)

33. Conformal flatness and the Schwarzchild interior solution (with S R Maity); J. Math. Phys. 20, 245 (1979)

34. Homogenoeous space-times of Godel type (with S Guhathakurta); Phys. Rev. D22, 802 (1980)

35. Viscous fluid interpretation of electromagnetic fields in general relativity (with S K Saha); J. Math. Phys. 22, 2237 (1981)

36. Rotating charged dust distributions in general relativity; J. Phys. A15, 831 (1982)

37. Viscous fluid interpretation of electromagnetic fields in general relativity II (with S K Saha); J. Math. Phys. 23, 2554 (1982)

38. Temperature dependent gravitational constant and black hole physics (with B Bagchi); Phys. Lett. B124, 168 (1983)

39. Dual interpretation of electromagnetic fields in general relativity (with S K Saha); Gen. Relativ. Gravit. 15, 611 (1983)

40. Is the universe near the state of maximal expansion? (with G Mukherjee); Mon. Not. R. Astron. Soc. 209, 353 (1984)

41. Physical approach to cosmological homogeneity (with B Modak); Phys. Rev. D31, 1807 (1985)

42. Domain walls of finite thickness in general relativity (with G Mukherjee); Phys. Rev. Lett. 59, 1504 (1987)

43. Inflation with arbitrary initial conditions; Class. Quantum. Gravit. 5, 225 (1988) 
44. Cosmic strings in general relativity; Phys. Rev. D41, 3041 (1990)

45. Theorem for non-rotating singularity free universes; Phys. Rev. Lett. 80, 645 (1998)

46. N K Dadhich and A K Raychaudhuri, Mod. Phys. Lett. 14, 2135 (1999)

47. A new theorem in relativistic cosmology; Mod. Phys. Lett. 15, 391 (2000)

48. Singularity free cosmological solutions with non-rotating perfect fluids; Gen. Relativ. Gravit. 36, 343 (2004)

\section{B. Books}

1. Theoretical Cosmology (Clarendon Press, Oxford; Oxford University Press, 1979)

2. Classical Mechanics - A Course of Lectures (Oxford University Press, India, 1983)

3. Classical Theory of Electromagnetic Fields (Oxford University Press, India, 1990)

4. General Relativity, Astrophysics and Cosmology (with S Banerji and A Banerjee) (Springer-Verlag, 1992)

5. Uchchataro Gatibidya (Higher Mechanics - in Bengali) (Paschim Banga Rajya Pustak Parishad, Kolkata)

6. Some outstanding problems in cosmology; in Gravitation and Relativistic Astrophysics: edited by Prasanna et al (World Scientific, 1984)

\section{Acknowledgements}

I am grateful to Naresh Dadhich for inviting me to make this contribution to the Puri conference, and also for providing me with a copy of his article 'A Lighthouse Falls', a memorial on Raychaudhuri, prior to publication. The article 'Solitary Pursuit' in The Telegraph by Parthasarathi Majumdar was helpful, too. My thanks go to Anita Ehlers and the excellent www-connections offered by IUCAA, through which I - though a computer illiterate - could establish fast contact with my former fellow students, notably Engelbert Schucking.

\section{References}

[1] A K Raychaudhuri, Arbitrary concentration of matter and the Schwarzschild singularity, Phys. Rev. 89, 417 (1953)

[2] A K Raychaudhuri, Relativistic cosmology, Phys. Rev. 98, 1123 (1955)

[3] There they say on p. 448: 'An important theorem for arbitrary models with incoherent matter has been discovered by A K Raychaudhuri in 1955. It rests on the Raychaudhuri equation for the scalar of expansion' 


\section{$J$ Ehlers}

[4] O Heckmann and E Schucking, in Gravitation: An introduction to current research (New York, 1962)

[5] J Ehlers, Contributions to the relativistic mechanics of continuous media, Gen. Relativ. Gravit. 25, 1225 (1993), Translation of the 1961 German original

[6] S W Hawking and G F R Ellis, The large scale structure of spacetime (Cambridge, 1973)

[7] A K Raychaudhuri, Theoretical cosmology (Oxford University Press, 1979) 